\title{
Topic index
}

Special Topics for ISOQOL Oral and Poster Sessions:

Cancer 5, 33, 40, 84

CAT IRT DIF 46

Chronic Illness 85

Communications 13

Condition Specific 34

Diabetes 88

Disparities 53

Family, Caregivers, and Social Support 24

Frontiers of Quality of Life Research 29, 36

Geriatrics 89

Health Policy and Resource Allocation 10
Lung Disorders/Pulmonary 57, 63

Mixed Methods 12

Multiple Sclerosis and Pain 6

Obesity 92

Pediatrics and Adolescents 93

Psychometrics 64

Qualitative Research 57

Response Shift 21, 106

Scale Development 71

Theory 75

Translation and Cultural Adaptation 78

Utilities 26,81 